\title{
Spectral wave modelling of Typhoon Krosa
}

\author{
A. V. Babanin ${ }^{1}$, T.-W. Hsu ${ }^{2}$, A. Roland ${ }^{3}$, S.-H. Ou ${ }^{4}$, D.-J. Doong ${ }^{5}$, and C. C. Kao $^{2}$ \\ ${ }^{1}$ Swinburne University of Technology, P.O. Box 218, Hawthorn, Victoria 3122, Australia \\ ${ }^{2}$ Department of Hydraulic and Ocean Engineering, National Cheng Kung University, Tainan 701, Taiwan \\ ${ }^{3}$ Institute for Hydraulic Engineering and Water Resources Management, Technische Universität Darmstadt, Rundeturmstr. 1, \\ Darmstadt 60483, Germany \\ ${ }^{4}$ Department of Environmental Resources Management, Tajen University, Pingtung 907, Taiwan \\ ${ }^{5}$ Department of Marine Environmental Informatics, National Taiwan Ocean University, Keelung 202, Taiwan
}

Received: 28 September 2010 - Revised: 30 November 2010 - Accepted: 1 December 2010 - Published: 16 February 2011

\begin{abstract}
This paper presents modelling the wave conditions in Typhoon Krosa prior to touching Taiwan in October 2007, with third-generation wave models of SWAN and WWM. The accuracy of the extreme wave measurement $H_{\text {max }}=32 \mathrm{~m}$ with significant wave height $H_{\mathrm{s}} \approx 24 \mathrm{~m}$ at the depth of $h=38 \mathrm{~m}$ is discussed first. It is concluded that the measurement does not appear faulty and is physically realistic. The numerical results are then analysed in order to examine the models' capability to reproduce the observed conditions. It is found that neither SWAN nor WWMII are able to hindcast the extreme measurement. Series of sensitivity tests are conducted for different numerical and diffraction schemes, and source functions. It is shown that, in the circumstances, the model performance only improves in response to the bottom-limited breaking formulation.
\end{abstract}

\section{Introduction}

In early October 2007, a category-4 Typhoon Krosa originated east of the Philippines in the Western Pacific and proceeded towards Taiwan. On 6 October, the highest ever wind-generated waves were recorded, with the trough-tocrest elevation for an individual wave of

$H_{\max }=32 \mathrm{~m}$

over a 10-min record with significant wave height of

$H_{\mathrm{s} \max }=23.9 \mathrm{~m}$.

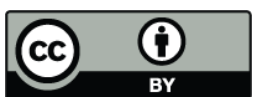

Correspondence to: A. V. Babanin (ababanin@swin.edu.au)
The track of Typhoon Krosa is shown in Fig. 1, with zoom in in Fig. 2. Three wave buoys were deployed in relatively close proximity. The Guishandao Buoy was positioned in the depth of $h=38 \mathrm{~m}$ between a small isle of Guishandao (Turtle Island) and the northeast tip of Taiwan and is indicated by the top cross symbol in Fig. 2. This is the buoy which recorded the maximal waves. Two other buoys were located further south, $25 \mathrm{~km}$ south of the Guishandao Buoy (this buoy is further called Suao Buoy) and $100 \mathrm{~km}$ south of the Guishandao Buoy (Hualien Buoy).

The three buoys are a standard long-term deployment by the Central Weather Bureau (CWB) of Taiwan. They are moored discus foam floats, $2.5 \mathrm{~m}$ in diameter, equipped with three-dimensional Watson accelerometers SHR-A1360-2A30/105. The data buoy was patented by the Republic of China (Taiwan) (patent number No. 087358, their period of validity runs from 1997 to 2016, see also Kao et al., 1999).

The three-component measurement of acceleration is essential in recovering height of extreme waves, as the discuss buoys with strapped-down accelerometers are known to exaggerate wave height (Bender et al., 2009). The buoys have been operational since 1997, and data are recorded for 10 min every hour sampled at $2 \mathrm{~Hz}$, thus providing 1200 values for each of the three accelerations and three angles. The vertical component is then double-integrated to obtain the surface elevation. This is a standard data-processing routine, see also Kao et al. (1999, 2003), Chiou et al. (2003) about its implementation in the CWB buoys. The buoy is directional, and Finite Fourier Series Method is employed for the derivation of directional wave spectra from the data buoys (Hsiao, 1994; Huang and Chen, 1998). It is based on the transformation functions for various measured properties of the buoy in the correlation between directional wave spectrum and cross spectrum that is presented by Isobe et al. (1984). 


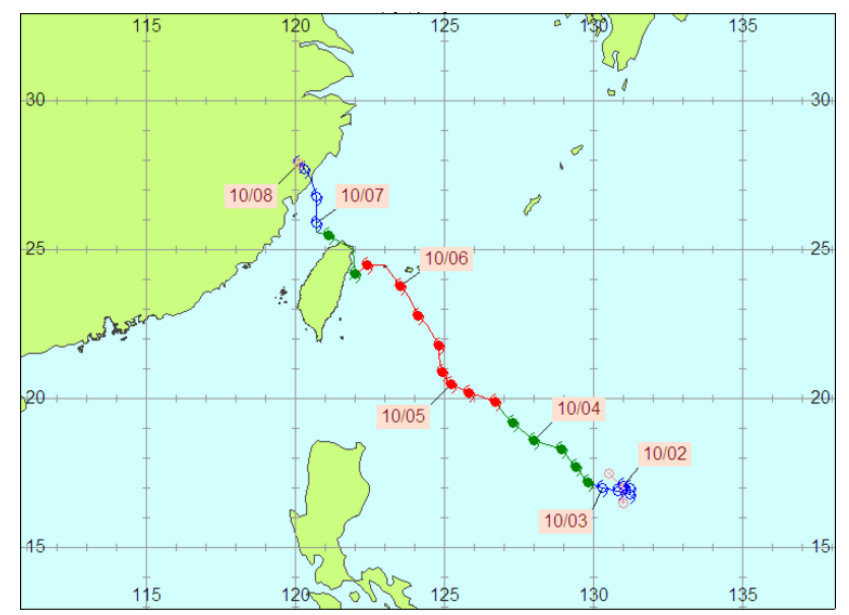

Fig. 1. Track of Typhoon Krosa (Central Weather Bureau (CWB), Taiwan).



Fig. 2. (a) Track of Typhoon Krosa near Taiwan. Locations of the Data Buoys are indicated with crosses.

All three buoys happened to be close to the path of the very centre of the Typhoon as shown in Fig. 2. The difference in the wave heights recorded by the three buoys, however, is striking. As shown in Fig. 3, the significant wave height $H_{\mathrm{s}}$ at the Suao and Hualien Buoys peaked one hour later compared to the Guishandao Buoy (note that $H_{\mathrm{S}}$ is sampled in one hour intervals), and was "merely" $11.7 \mathrm{~m}$ and $10.1 \mathrm{~m}$, respectively.

In the present paper, an attempt is made to hindcast the wave conditions off the northeast coast of Taiwan by means of the most up-to-date third-generation wave spectral models. Since the issue of measurements being realistic is central to the argument, it is addressed in the next Sect. 2. The hindcast setup and sensitivity study are presented in Sect. 3. The final Sect. 4 is dedicated to discussion of the sensitivity tests and conclusions which can be made with respect to the modelling of such unique and extreme environmental situations.

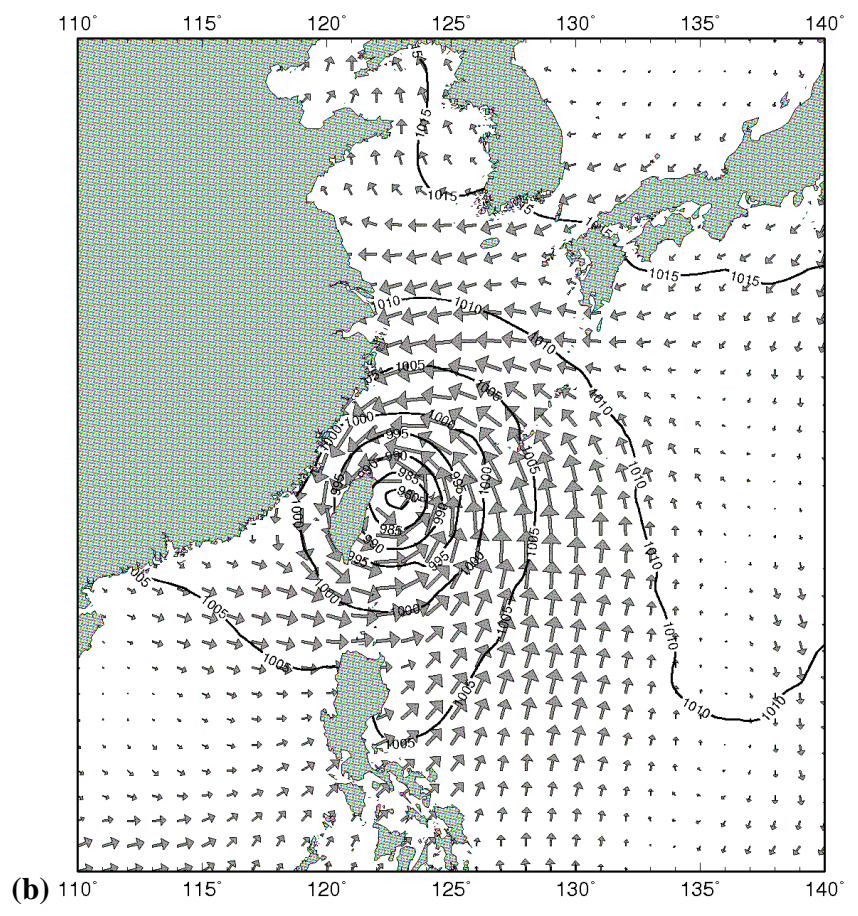

(c)

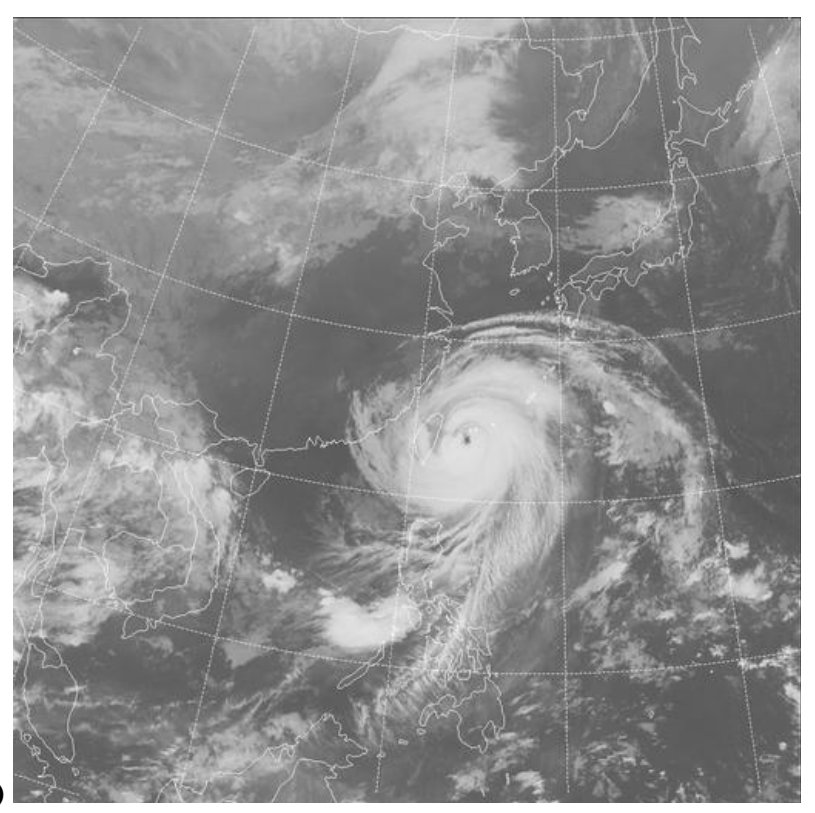

Fig. 2. (b) Wind field provided by CWB (6 October 2009, 13:00). The maximal mean wind speed near the typhoon centre is $51 \mathrm{~m} \mathrm{~s}^{-1}$. The maximal gust wind is $63 \mathrm{~m} \mathrm{~s}^{-1}$. (c) Satellite image of typhoon Krosa on 6 October 2009, 07:00.

\section{The measurements}

The issue of accuracy of the unique extreme measurements is critical for the discussions presented here and for the modelling attempt, and therefore has to be addressed first. In other words, can we trust the measurements being realistic and were the waves indeed as high as it shows? 

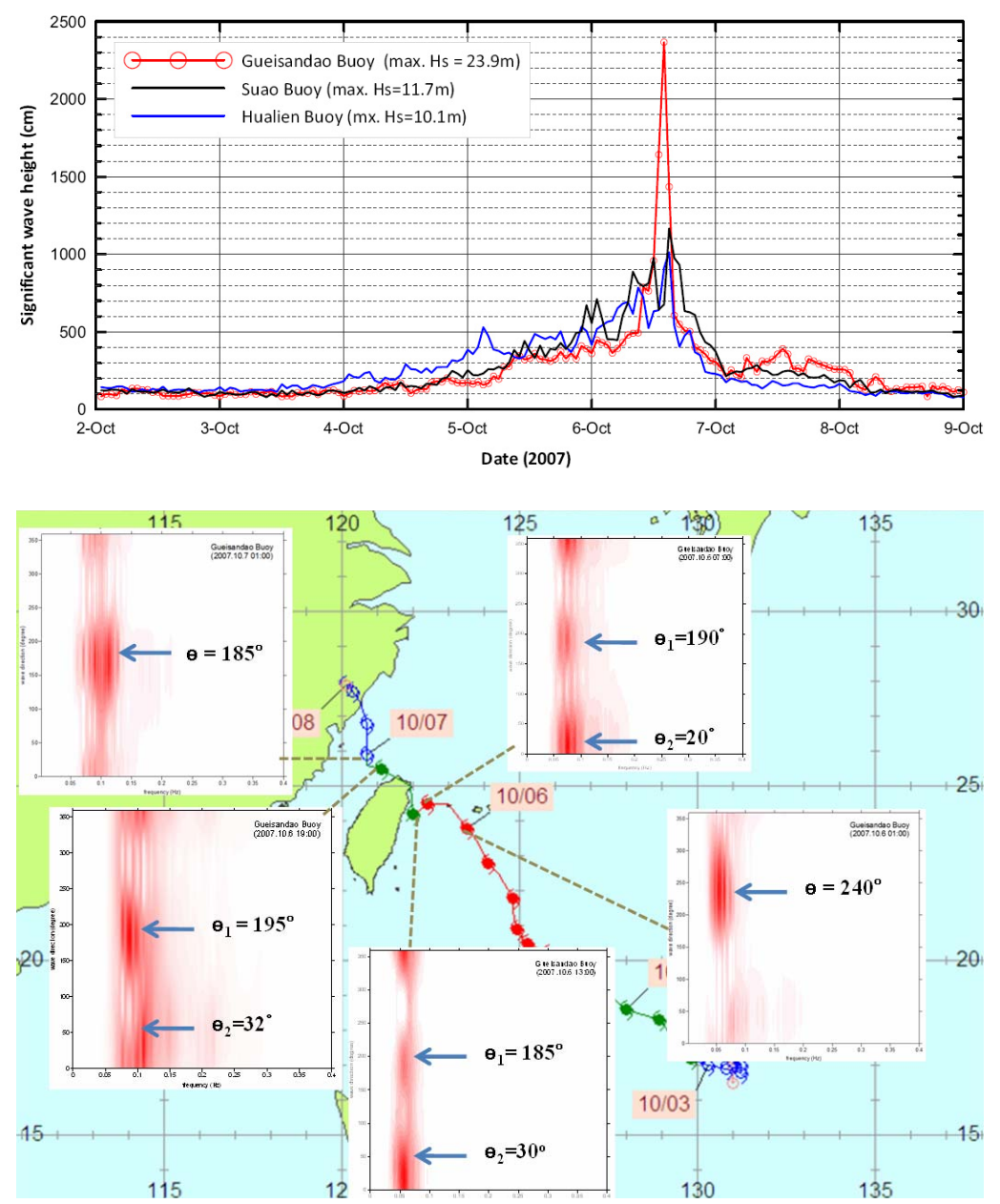

Fig. 3. Top: significant wave height recorded on hourly basis between 2 and 9 October at the Guishandao, Suao and Hualien Buoys. Bottom: directional wave spectra during Typhoon Krosa.

The answer appears positive. Liu et al. (2008) and Doong et al. (2009) discussed the instrumentation performance and did not find any faults. They thoroughly analysed data and did not reveal any inconsistencies in the wave records either.

Indeed, apart from some drift in the Guishandao Buoy's position, nothing unusual was observed. The drift was minor and the buoys gradually travelled some $350 \mathrm{~m}$ overall during 6 October as detected by GPS positioning. The 91-m-long mooring remained intact, as subsequent inspection showed. Generally, the inspection revealed some surface damage to the buoy, but nothing major, and even a vane anemometer on the top of the Buoy's mast survived.

Analysis of the data demonstrated that the accelerometers worked without faults throughout, and continued working after the Typhoon passed. Figure 3 demonstrates that records of the Guishandao Buoy are fully consistent with those by Suao and Hualien Buoys, except for the peak of the Typhoon. The extreme significant wave height measured at the peak is not a spike in the record, but is preceded by the hour when $H_{\mathrm{s}}$ was rising and followed by the hour when $H_{\mathrm{S}}$ was decreasing.
In the figures and the text, all times are local. That is, at 11 a.m. on 6 October, the Guishandao Buoy recorded the $10 \mathrm{~min}$ significant wave height of $9.6 \mathrm{~m}$, the same as the Suao Buoy. At 12:00 noon, this height grew to $16.3 \mathrm{~m}$, essentially exceeding the measurements by Suao and Hualien Buoys, then to $23.9 \mathrm{~m}$ at $1 \mathrm{p} . \mathrm{m}$. (see Eq. 2, the peak), after that time it started rapidly dropping. At 2 p.m., the Guishandao Buoy measured $H_{\mathrm{s}}=14.2 \mathrm{~m}$, just $2.5 \mathrm{~m}$ higher than the Suao Buoy, and at 3 p.m. $H_{\mathrm{s}}=6.1 \mathrm{~m}$, well below the Suao Buoy and comparable with the background measurements of the relatively distant Hualien Buoy.

During this time, the Typhoon was moving straight head on the Guishandao Island and the Guishandao Buoy behind it, between 11 a.m. and 1 p.m. This is the period when the wave height recorded was sharply growing at the Guishandao Buoy. At 3 p.m., as the Typhoon diverted to the west and bypassed the Turtle Island, the wave height dropped down.

Thus, the records of the significant wave height by the Guishandao Buoy appear consistent with the records conducted by the other Buoys in area and with the track of 
the Typhoon. Scrutinising individual waves recorded and the wave spectra obtained provides further material for analysis.

In Doong et al. (2009), it is mentioned that for the maximal significant wave height the mean wave period is $13.8 \mathrm{~s}$ which, in $h=38 \mathrm{~m}$ depth, converts into wavenumber $k_{\text {mean }} \approx 0.027 \mathrm{rad} \mathrm{m}^{-1}$ and wavelength of $\lambda_{\text {mean }} \approx 230 \mathrm{~m}$. Thus, $k h \approx 1$, which signifies finite depth, but not shallow waters. In the finite-depth environment, the wave dynamics should be affected by the bottom proximity, compared to the deep-water, but is not restricted to the non-dispersive wave medium. The phase speed of quasi-linear 230-m-long waves at $k h \sim 1$ would be $c \sim 17 \mathrm{~m} \mathrm{~s}^{-1}$, which is still significantly faster than their group velocity of $C_{\mathrm{g}} \sim 13 \mathrm{~m} \mathrm{~s}^{-1}$. Thus, the media is still dispersive, but conditions for the modulational instability are marginal, and the linear focusing, finite-depth and diffraction effects, or a combination of them, are a likely cause for the observed extreme.

In any case, the mean steepness of

$\varepsilon_{\text {mean }}=\frac{H_{\mathrm{s}}}{2} k_{\text {mean }}=0.33$

is very high for field waves, but not impossible. If the modulational instability is active, such steep-in-the-mean waves should develop modulation with a relatively low number of waves in the group (e.g. Babanin et al., 2010), which is what the record shown in Liu et al. (2008) and Doong et al. (2009) demonstrates. In a quasi-twodimensional wave train with the mean steepness of (3), a wave breaking should develop within approximately 20 wave periods, leading to the wave having individual wave steepness of

$\varepsilon=\frac{H}{2} k=0.44$

(Babanin et al., 2007). This is exactly the steepness of the highest wave with $H=32.3 \mathrm{~m}$ (1) shown in the record of some 30 waves by Liu et al. (2008) and Doong et al. (2009). It should be also noted that the directional wave spectrum at the peak of the Typhoon recorded by the Guishandao Buoy became very narrow (Doong et al., 2009, see also Fig. 3), an important condition for the modulational instability to develop (Janssen and Herbers, 2009; Onorato et al., 2009a, b; Waseda et al., 2009; Babanin et al., 2011).

At the same time, conditions for the wave focusing were also appropriate. The linear frequency focusing is always an option in dispersive environments (see e.g. a detailed study by Rapp and Melville, 1990), as is the amplitude focusing (e.g. Pierson et al., 1992). With the background mean steepness of Eq. (3), the superposition would not require many components, perhaps just two, for the outlier like in Eq. (1) to occur (see more on this topic in Babanin et al., 2011).

Most important possibility in the circumstances was also directional focusing (see e.g. Fochesato et al., 2007; Babanin et al., 2011). This is a three-dimensional phenomenon, and the finite-depth conditions can potentially magnify or even trigger the effect under appropriate wavelength/direction, water-depth and bottom-topography situations. Provided two wave systems with close wavelengths are present, which they were (see Fig. 3, bottom), and the Buoy is in the focal point of the angular deflection, the directional focusing can actually double the significant wave height rather than just produce single extreme events like the modulational instability or the dispersive focusing.

If the directional focusing was indeed happening, and the buoy was being approached by a series of concave/convex converging/diverging wave crests/fronts, it is difficult to say what direction or directional distribution the Buoy's system, designed for longcrested directional components would output. It is hard to imagine, however, that an artifact would be a directional spectrum consisting of two wave systems travelling in nearly opposite directions, i.e. at nearly-south $190^{\circ}$ and nearly-north $20^{\circ}$. And such was the measurement of the Guishandao Buoy for at least $12 \mathrm{~h}$, including $6 \mathrm{~h}$ before and $6 \mathrm{~h}$ after the extreme event described by Eqs. (1)-(2) according to Doong et al. (2009).

Therefore, although the directional focusing would allow us to explain not only the extreme individual event, but also the extreme significant wave height, this reason cannot be stated responsible with any certainty either. This is particularly true as the systems of opposing waves of similar magnitude are known to bring about very dangerous seas, but not the doubling the average wave height (see, for example, investigation of the catastrophic storm during the 1998 Sydney-to-Hobart yacht race; Greenslade, 2001).

In summary, we have to conclude that the measurements do not appear to be faulty in any respect, and are physically realistic in principle, although are certainly extreme beyond expectation. We must say that not all technical issues which may have affected the buoy's reading in the circumstances are well clear. For example, the anchored buoys cut through the sharp crests of the waves (Liu et al., 2008), and accelerometer buoy measurements tend to smooth the crest accelerations (e.g. Babanin et al., 1993; Liu and Babanin, 2004), that is to underestimate the height of large skewed waves. Therefore, distortions of the wave shape recorded by the accelerometer buoy are certainly possible, but magnifying the wave height, yet alone consistent magnifying of the significant wave height by the factor of two or so over the period of a few hours, is certainly impossible, particularly as two other buoys of the same construction and using the same instrumentation did not exhibit any peculiarities in the same circumstances.

Physical cause of such extreme wave condition is the main unknown, and is of the main interest from the point of view of wave forecast in extreme environment. Cavaleri (2009) in his recent review of the state of the art of wave modeling demonstrated that it is the extreme situations which are mainly failed by the modern models. Study of Cavaleri (2009) was only conducted for deep-water 
situations, but his conclusions are even more applicable to the finite depths where additional uncertainties and physics come to play. Therefore, in the next Section we will verify ability of the existing third-generation wave models to reproduce the observations.

\section{Numerical modeling of Typhoon Krosa}

\subsection{The wind fields}

Most typhoons that occur around Taiwan Island are tropical cyclones frequently formed in the northwestern Pacific Ocean. Severe typhoon events often produce fields of strong winds, storm surges and large waves that cause coastal damages. In late September 2007, an atmospheric system was generated east of the Philippine which, as a result of rapid intensification on 2 October, was suddenly upgraded as a typhoon named Krosa. The track of Typhoon Krosa hovered in a small loop over the northeastern coastal waters of Taiwan (Fig. 1) and touched Taiwan on 6 October. The translation speed of Typhoon Krosa before approaching Taiwan Island was about $14 \mathrm{~km} \mathrm{~h}^{-1}$ and then reduced to $11 \mathrm{~km} \mathrm{~h}^{-1}$ during its impact on the land. According to the record of CWB, the lowest central pressure was $925 \mathrm{hpa}$, the maximal wind speed was $52 \mathrm{~m} \mathrm{~s}^{-1}$ (101 knots), and the largest radius reached $120 \mathrm{~km}$.

The wind fields used in this paper were obtained from CWB and have a resolution of $15 \mathrm{~km}$ in geographical space and a temporal resolution of one hour. The data were interpolated on the numerical mesh (see Fig. 5 below) using linear interpolation in time and space. Since the numerical mesh has a high resolution and the greatest integration time step of the wave model $(600 \mathrm{~s})$ is much less than the time increment for which the wind field is available, there are no serious interpolation errors anticipated. In Fig. 4, the quality of the wind fields is checked by comparing to the buoy measurements at the three buoy locations. We notice that the modelled wind fields are in good agreement with the buoy measurements at Guishandao and Suao stations.

\subsection{The wave modelling}

To evaluate performance of the spectral wave models when modelling such extreme waves, as measured at Guishandao Island during Typhoon Krosa, WWMII (Wind Wave Model II; Roland, 2009) and SWAN (Simulating WAves Nearshore; Booij et al., 1999) models were used in this study. WWMII model is the second version of WWM (Hsu et al., 2005). Both models have been successfully used in different studies, including forecast of typhoon (see e.g. Ou et al., 2002; Hsu et al., 2005) or hurricane waves in the US (Roland et al., 2005). Results of these studies showed the feasibility of using these two spectral wave models for the simulation of extreme waves, at least up to $15 \mathrm{~m}$ of height.
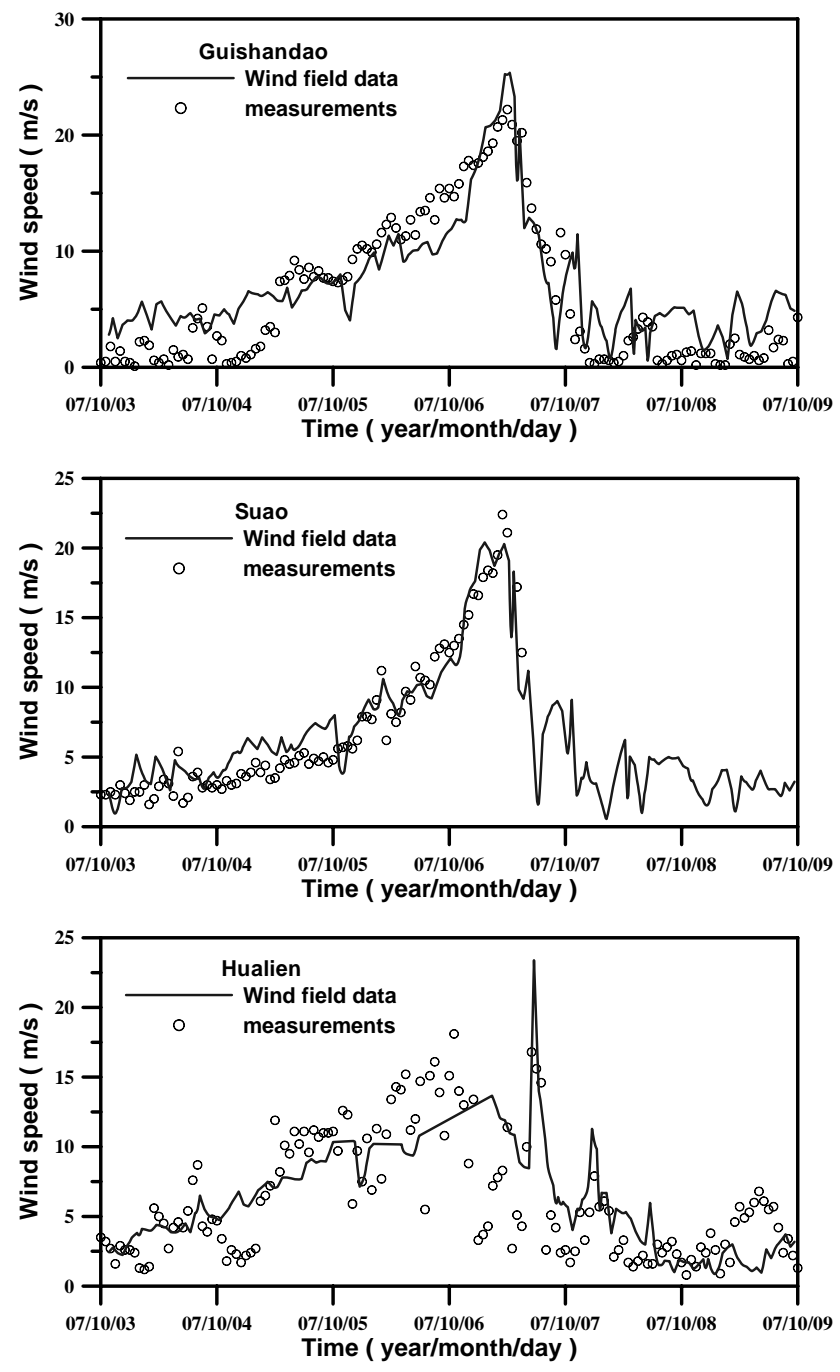

Fig. 4. Wind velocity measured by the buoys and hindcasted by CWB.

For the simulation, an unstructured mesh has been produced, with higher resolution in the nearshore region and around Guishandao Island and the other buoy positions (Fig. 5). The resolution of the mesh varies from $42 \mathrm{~m}$ in the vicinity of Guishandao Island to $100 \mathrm{~km}$ far away of the region of interest.

The bathymetry and the location of the data buoys that were considered here for comparison purposes, like Hualien and Suao Buoys, are given in Fig. 6. It should be noted that the bathymetry was interpolated from different databases. We used the ETOPO1 dataset (Amante and Eakins, 2009) for the part far from Taiwan and a 500-m bathymetry is available in the vicinity of Taiwan. In the vicinity of the buoys Sonar soundings have been made before the deployment of a buoy which have been used too. These databases were kindly provided by the COMC (Coastal and Ocean Monitor Center), National Cheng Kung University, Taiwan, for this 


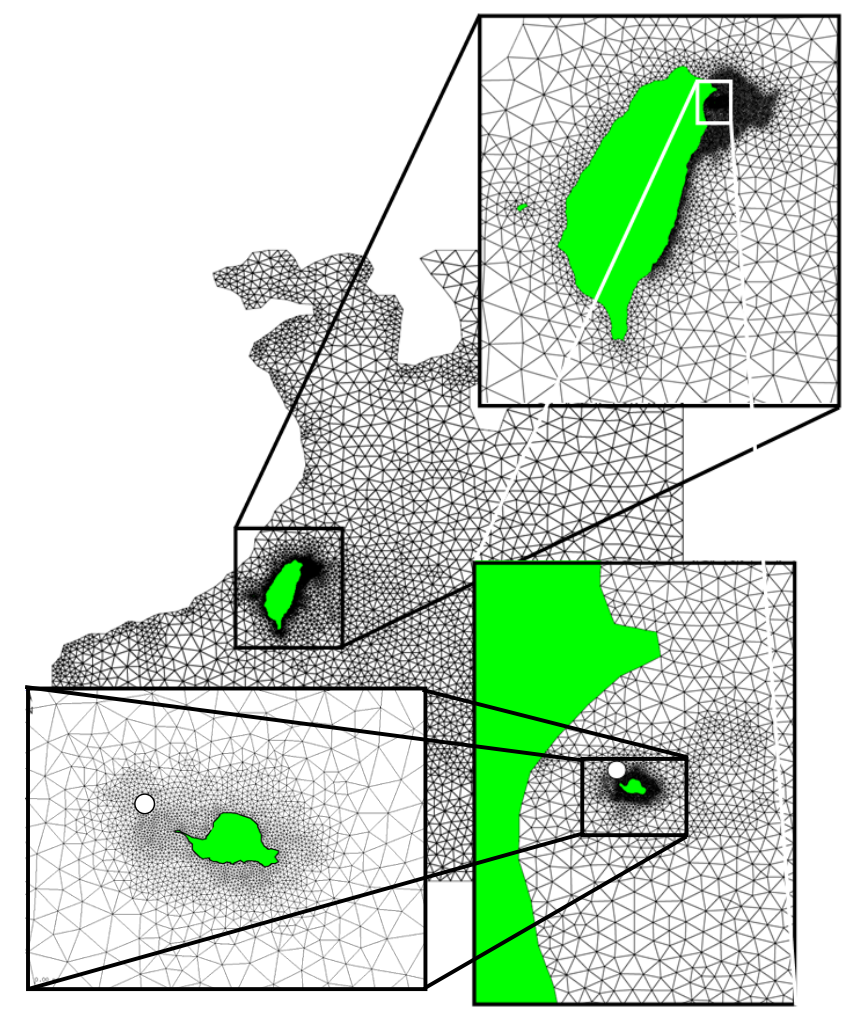

Fig. 5. Configuration of the unstructured mesh around Guishandao Island.

study. From the depth distribution in Fig. 6, it can be seen that the Guishandao Buoy is located just over an underwater shoal.

WWMII solves Wave Action Equation (WAE) with the aid of the fractional step method (Yanenko, 1971). The multidimensional problem is split into parts (see e.g. Hsu et al., 2005 or Roland, 2009) and the solution is advanced by solving each part successively. WWMII uses, for the advection part in geographical space, numerical schemes that work on unstructured meshes by applying residual distribution schemes (see e.g. Abgrall and Mezine, 2006). The schemes are monotone and are ranging from linear implicit 1 st order up to nonlinear explicit 2 nd order spacetime schemes, the spectral part advection is solved as in WAVEWATCHIII Tolman (2002) using the Ultimate Quickest scheme.

The SWAN model in its latest version (Zijlema, 2010) works also on unstructured meshes and was used here for comparison purposes. In contrast to WAVEWATCHIII or WWMII, in SWAN the whole equation is solved at once. The source terms are linearised, the advective terms are solved using lower-order schemes on unstructured meshes (for details, see Zijlema, 2010) and the spectral part is solved using linear methods up to 2 nd order. In the numerical simulation, we tested the models using the default

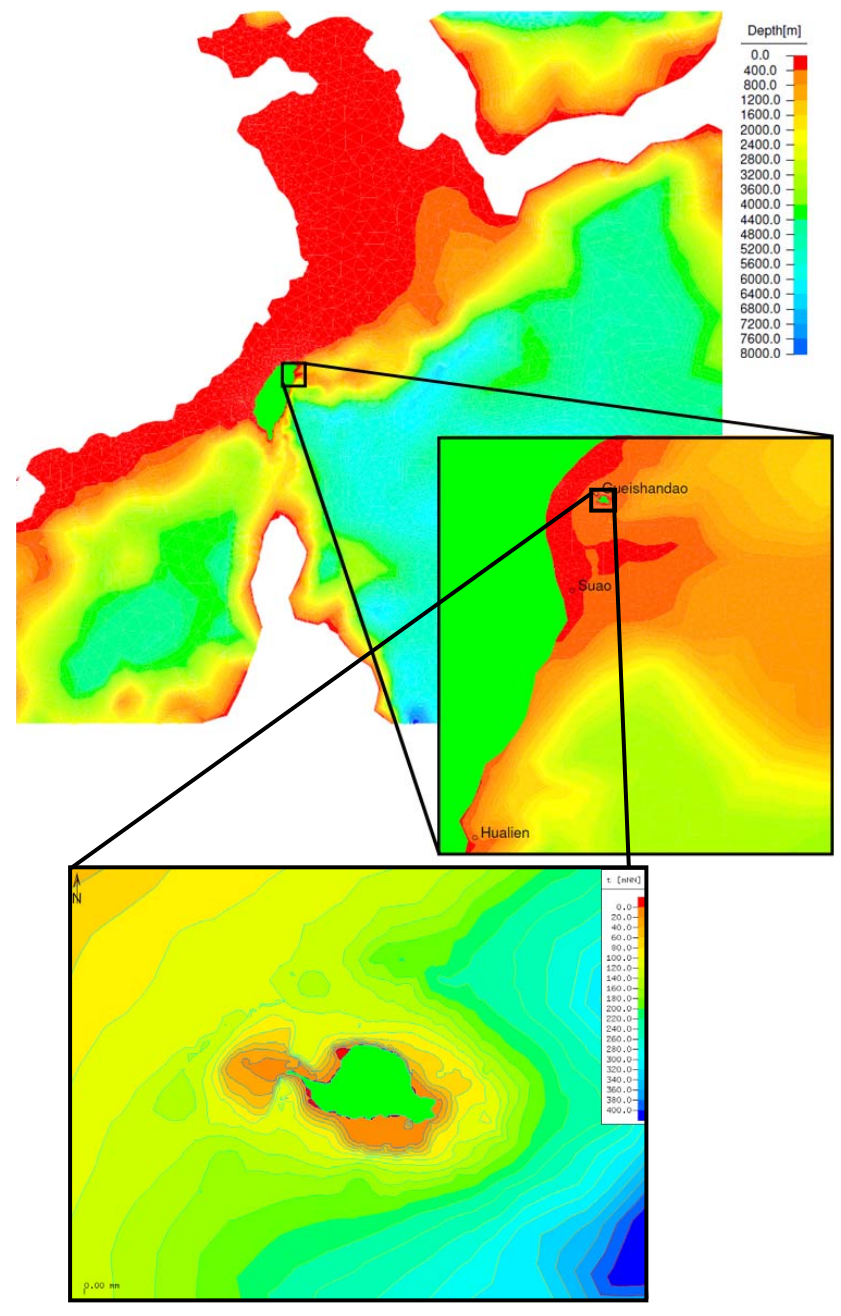

Fig. 6. Depth distribution in the modelling region around Guishandao Island.

formulation of SWAN model, which is defined in Booij et al. (1999) and mostly called the Cycle 3 formulation. Other simulations have been carried out using the formulation which follows Komen et al. (1994) for wind input und dissipation (so-called Cycle 4 formulation). In WWMII, however, we also used the latest formulation of Bidlot et al. (1997), which resembles the ECMWF wave-forecasting system with respect to the formulation of the source terms. The shallow-water effect was modeled as in SWAN model using the wave-breaking term in shallow water according to Battjes and Janssen (1978). For the nonlinear wavewave interactions in shallow water, the Lumped Triad Approximation (LTA) of Eldeberky (1996) is parameterised as in the SWAN model code.

The simulations have been carried out using lower-order schemes that were mostly utilised in spectral wave models such as SWAN or WAM. The discretisation in directional space $\Delta \theta$ was $10^{\circ}$, the relative frequency resolution $\Delta \sigma / \sigma$ 
was 1.1. The frequency bandwidth was set in both models from $0.04-1.0 \mathrm{~Hz}$. The integration time step was set to $600 \mathrm{~s}$ as often used in spectral wind-wave modeling in operational forecasting.

Beside this "operational" setup, the effect of the numerics on the results have been investigated by using 2 nd order monotone schemes (Roland et al., 2009) in geographical space combined with the 3rd order Ultimate Quickest scheme for spectral advection in the WWMII. The source terms have been integrated for this run using a three-step Runge-Kutta method. In order to reduce any effects due to the big time steps of $600 \mathrm{~s}$ and to reduce possible influence of the "Actionlimiter" (see e.g. Tolman, 2002) on the results the integration, time step was reduced to $60.0 \mathrm{~s}$ in some simulations.

\subsection{Results and discussion}

Results of the numerical simulations are shown in Fig. 7 for all the three buoy locations. The wave heights of Eq. (2) measured at Guishandao Island could not be reproduced by any of the investigated simulation runs. For the other two buoy locations, the wave height is also underestimated in the vicinity of the peak values during Typhoon Krosa. It is evident that the underestimations are considerable but they are much smaller if compared with those at Guishandao Island and most likely are caused by other reasons.

Variation of the results due to the numerical and physical settings is marginal with respect to the large gap between measured and simulated significant wave heights at Guishandao Island. This indicates that neither deepwater source-term formulations nor numerical schemes are the reason for the failure of the spectral wave models during this event. The fact that hindcasted waves are underestimated at all three locations points out to some principal limitations of wave models, rather than to some local peculiarity of the Guishandao Island environment only.

Figure 8 shows refraction characteristics in the vicinity of the Guishandao Buoy during the passage of the Typhoon at the time when the significant wave height reaches its peak value. The focusing of wave energy over the shoal in the vicinity of the buoy can be clearly seen.

It is well known that the spectral wave models can predict strong focusing of wave energy over a submerged shoal. Many investigations which used spectral wave models like SWAN or WWM (e.g. Holthuisen et al., 2003; Roland, 2009) could reproduce the wave height distribution for the laboratory experiment of Vincent and Briggs (1989). Mostly, spectral wave models, when at least second-order schemes are used, even overestimate the maximal measured amplification factor compared to the Vincent and Briggs in this case. The reason for this overestimation lies in the absence of diffraction. If phase-decoupled diffraction approximation used (e.g. Holthuijsen et al., 2003) the results fit the measurements better.

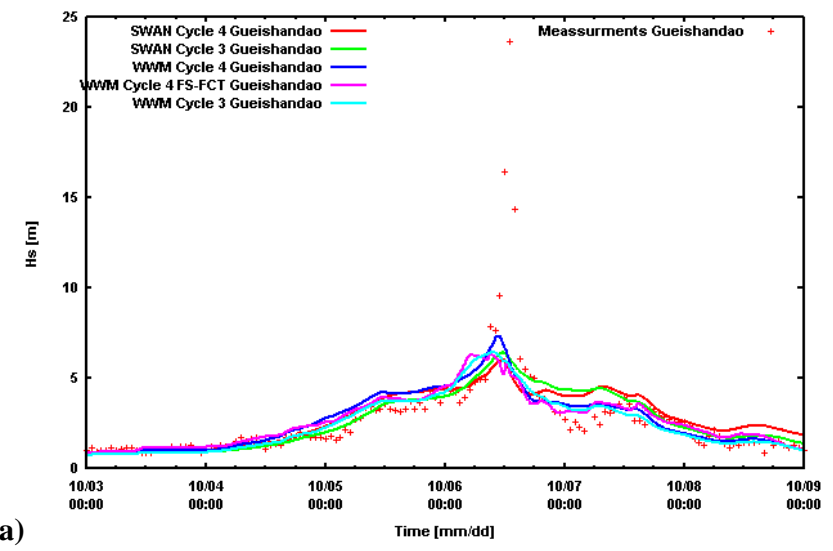

(a)

(b)



(c)



Fig. 7. Simulation results and measurements for the three buoy locations. (a) Guishandao Island; (b) Suao Buoy; and (c) Hualien.

Coming back to the situation at Guishandao Island, where wave focusing over the shoal was obviously taking place, the question that remains open is why in this situation no amplification of the waves can be observed in the models. Since we have already investigated the influence of deep-water physics, time steps and numerical schemes without much success, formulations/parameterisations of the shallow-water source terms remain an open issue. 


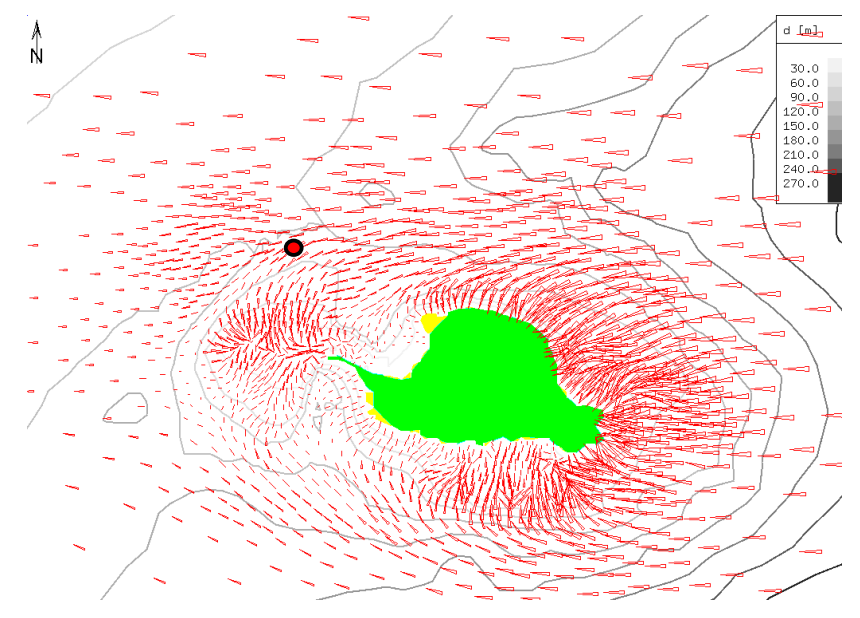

Fig. 8. Refraction characteristics in terms of mean wave direction obtained using WWMII during the measured peak wave height at Guishandao Island (red dot shows the buoy location).

In order to investigate sensitivity of the wave-breaking source term in shallow water, we conducted an academic test by integrating using a steady wind field with velocity of $30 \mathrm{~m} \mathrm{~s}^{-1}$ coming from the east of Guishandao Island. We integrated WAE by using WWMII and SWAN model until steady state was reached. As it can be seen from Fig. 9, there is clearly an accumulation of wave energy in the focusing region but there is no amplification at all, by comparison with the wave heights in deep water. In fact, the opposite is true: here the island shelters the region in the lee side. This indicates that there is no amplification of the incoming wave energy over the shoal, the sheltering effect of the islands and the contribution of the shallow-water wave-breaking term do not allow any kind of amplification of wave energy in this region if using 3rd generation spectral wave models. The results are generally similar, when using either WWMII or SWAN.

In the next computational run, we modified the wavebreaking source term, in particular we changed the criterion according to which the maximum wave height is estimated, the so-called "wave breaking criteria". This breaking criterion acts as a hard limit on the solution as it does not allow the existence of waves higher than those determined by it. In the simplest case the wave breaking criterion is defined as a fraction of the water depth and it is often set to 0.73 . This is used as a default in the SWAN model.

Here, we allowed the wave to be up to twice that size by setting the wave breaking criterion to 1.5 . The wave dissipation function by Battjes and Janssen was designed for the bore wave breaker on gentle slopes, where it indeed gives excellent results. It does not necessarily hold on steep slopes, for plunging breakers or in such condition as observed and indicated by the modeling results here. One could argue that because the waves are coming from opposite
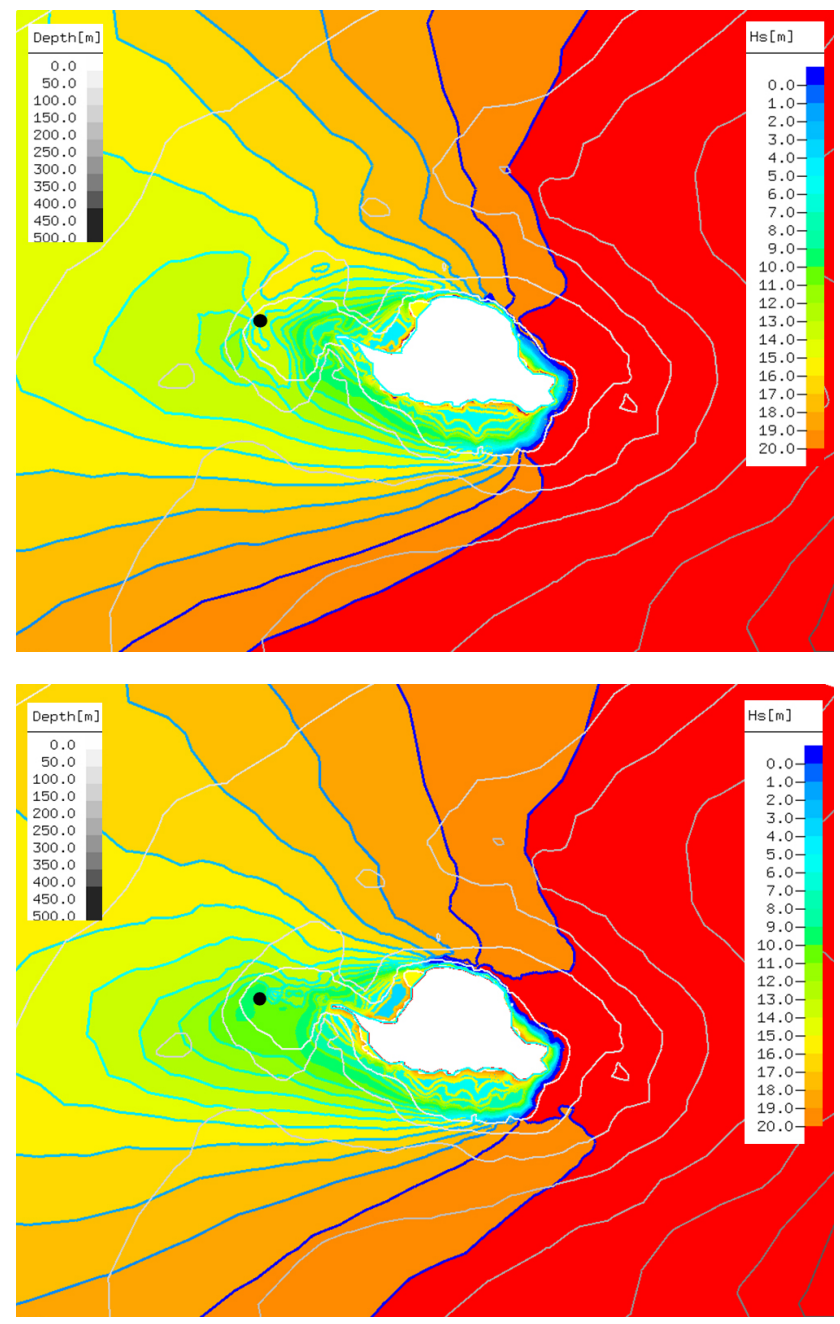

Fig. 9. Refraction characteristics in terms of significant wave height around Guishandao Island based on steady easterly wind of $30 \mathrm{~m} \mathrm{~s}^{-1}$ (top: SWAN, bottom: WWMII; * black dot indicates the position of the wave buoy). Grey scale for the contours indicate the water-depth isolines. Colour scale on the right describes the significant wave height.

directions, greater values of the breaker criterion are possible since during the collision of these waves, coming around the island, they may be amplifying each other and so the fixed limit of 0.73 , which is mostly used in spectral wave modeling and therefore also in this study by default, may not be directly applicable. As an example, for standing waves the limiting steepness can be $50 \%$ or more higher than that indicated by Eq. (4) for progressive waves (e.g. Schwartz and Fenton, 1982, see also Babanin, 2009, Sect. 5.1.2, for a discussion).

In Fig. 10, results of using both WWMII and SWAN are shown. Both models showed that changing the default breaking coefficient had a significant impact on wave energy in the vicinity of the buoy. Compared to the influence of the numerics of the model and the physics of the source terms, which have been shown before, this was the most 

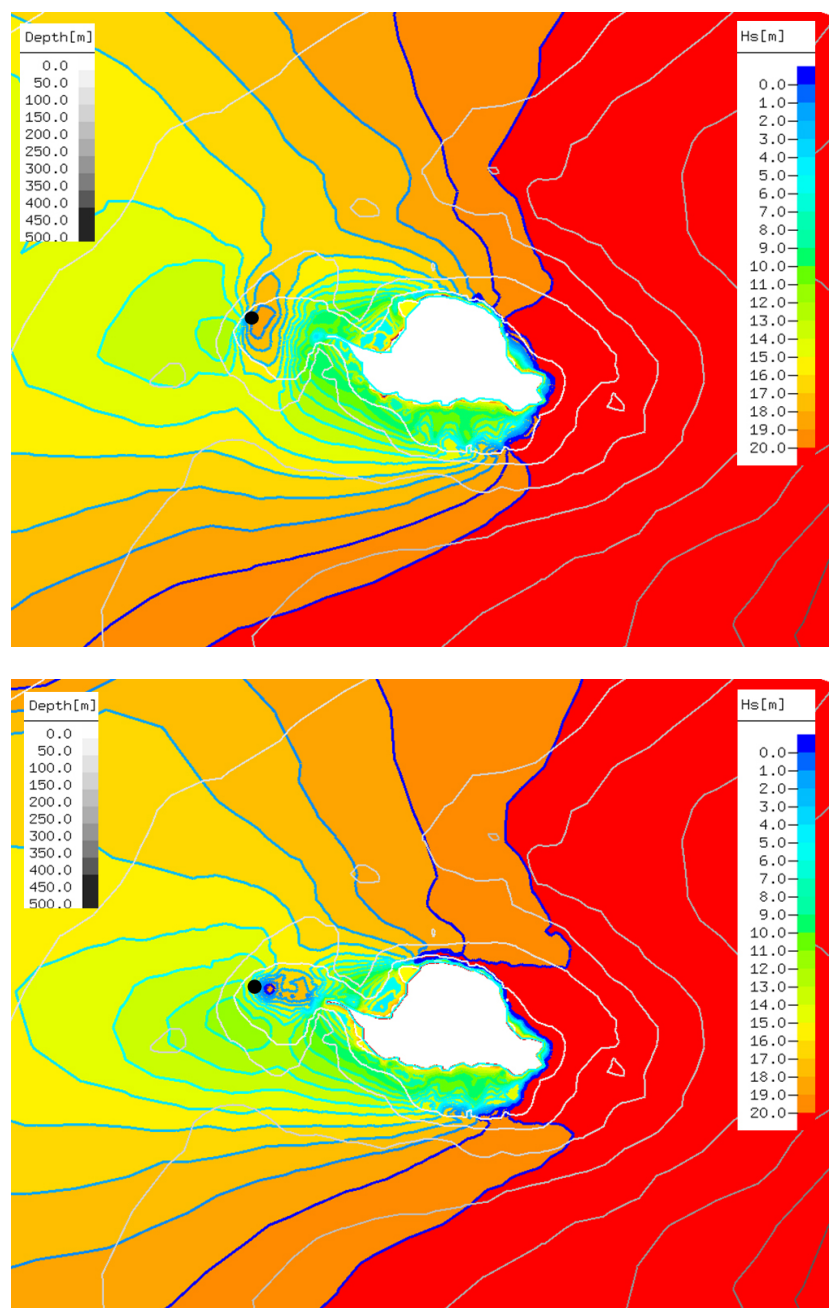

Fig. 10. As in Fig. 9, for constant wave-breaking coefficient of 1.5.

sensitive parameter in this study. Especially, it must be emphasised that the difference of the results using the modified breaker coefficient have been the largest at the location of the Guishandao buoy, which is a clear indication that the assumption of a constant breaking coefficient of 0.73 may not suitable in this situation.

It can thus be concluded that, apart from this moderately promising result with respect to the finite-depth wavebreaking criterion, this study is discouraging in terms of performance of the 3rd generation spectral wave models in such extreme situation. On the other hand, it is a great motivation for future research on such topics as wave breaking for strongly pronounced cross seas that are often occurring at focusing points.

Last but not least, we investigated the effect of phasedecoupled diffraction approximation according to Holthuijsen et al. (2003). Since the bottom slopes in the investigated region are very steep, wave diffraction will affect the wave

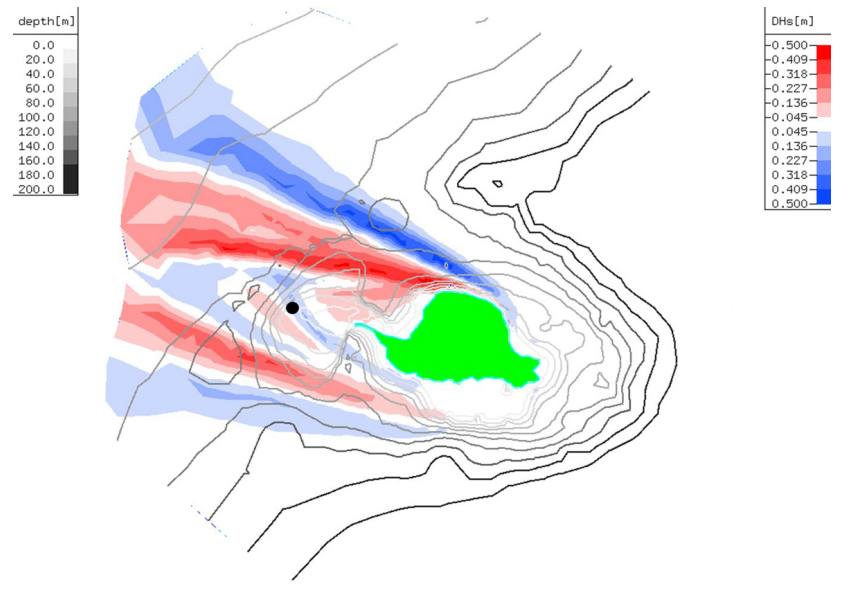

Fig. 11. Difference plot using phase decoupled diffraction. Red colour: enhancement of wave energy due to diffraction. Blue colour: reduction of wave energy due to diffraction (black dot indicates the position of the wave buoy).

evolution. In order to quantify this effect within the phaseaverage description we used a time step of $60 \mathrm{~s}$ and a directional resolution of $5^{\circ}$ in combination with the higherorder schemes for the left-hand side of WAE. It can be seen that there is a variation of around $0.5 \mathrm{~m}$ in the vicinity of the steep slope at the northern and the southern parts of the island, but in the vicinity of the buoy, where the strong amplification occurred, there is nearly no variation of the wave height due to wave diffraction (Fig. 11).

\section{Conclusions}

SWAN and WWM models were used to hindcast the giant waves observed during the passage of Typhoon Krosa at the northeastern tip of Taiwan in the vicinity of Guishandao Island. Both models incorporate recent formulations for the source-term balance. However, the models fail to hindcast the wave evolution during Krosa at Guishandao Island and the strong amplification of wave energy in the vicinity of the measurement buoy. Interesting to remark is that, for the other two buoys Suao and Hualien, an underestimation of wave energy also occurs, but to a much lesser extent compared to hindcasted and measured waves at Guishandao. The underestimation at these two other buoy locations, however, has clearly other reasons, e.g. the quality of the wind field or understanding of the generation and decay of typhoon waves. For the location of the Guishandao buoy, on which our study is concentrated, the sensitivity testing indicates that the reason can be the formulation of the finitedepth breaking source term, which in the case of a strongly bimodal directional setup, appears to dissipate too much wave energy. 
Physically, it is likely that the main reason for the generation of these giant waves is directional focusing which can lead to an amplification of up to a factor of two (see e.g. Fochesato et al., 2007; Babanin et al., 2011). If combined with shoaling effects, the amplification of the incident waves up to a factor of 2.5 can occur as has been observed in the laboratory (Vincent and Briggs, 1989) and has been reproduced by numerical modelling using spectral wave models (see e.g. Holthuijsen et al., 2003; Roland, 2009). Such amplification, however, did not appear to happen in the presented simulations. Variation of the numerical schemes, spectral discretisation and integration time step showed that the results for the undertaken simulations are not sensitive to the numerical features of the models either. The largest influence on the results was due to variation of the constant of the shallow-water wave-breaking criterion, which led to a strong enhancement of the wave height in the vicinity of the buoy.

As discussed in the Introduction, potential physical processes other than shoaling, which can lead to high waves in dispersive environments, are modulational instability and linear focusing. The phase-average models are not able to simulate the former, and under the circumstances, if it were not the likely the cause of the events anyway. Other non-linearities, such as those given through the source terms like the $\mathrm{S}_{\mathrm{n} 14}$ term (Quadruplet Interactions) and the $\mathrm{S}_{\mathrm{n} 13}$ term (Triad Interaction), are also conservative and cannot be responsible for the growth of significant wave height and therefore the energy. Frequency and directional focusing, which can be simulated using this model class, was apparently not up to a magnitude in the model as would be necessary to lead to the observed increase of wave energy. Therefore, the third-generation spectral wave models, in their actual formulation, cannot close the gap between measured and hindcasted waves heights for this event at the location of the Guishandao Buoy.

Acknowledgements. AVB gratefully acknowledges financial support of the Australian Research Council and Woodside Energy Ltd through the Linkage Grant LP0883888 and of the Australian Research Council through the Discovery Grant DP1093349.

Edited by: E. Pelinovsky

Reviewed by: P. Liu and two other anonymous referees

\section{References}

Abgrall, R. and Mezine, M.: Construction of second order accurate monotone and stable residual distribution schemes for unsteady flow problems, J. Comput. Phys., 188(2003), 16-55, 2006.

Amante, C. and Eakins, B. W.: ETOPO1 1 Arc-Minute Global Relief Model: Procedures, Data Sources and Analysis, NOAA Technical Memorandum NESDIS NGDC-24, 19 pp., 2009.

Babanin, A. V.: Breaking of ocean surface waves. Acta Physica Slovaca, 59, 305-535, 2009.
Babanin, A. V., Verkeev, P. P., Krivinskii, B. B., and Proshchenko, V. G.: Measurements of wind waves by means of a buoy accelerometer wave gauge, J. Phys. Oceanogr., 4, 387-393, 1993.

Babanin, A. V., Chalikov, D., Young, I. R., and Savelyev, I.: Predicting the breaking onset of surface water waves, Geophys. Res. Lett., 34, L07605, doi:10.1029/2006GL029135, 2007.

Babanin, A. V., Chalikov, D., Young, I. R., and Savelyev, I.: Numerical and laboratory investigation of breaking of steep twodimensional waves in deep water, J. Fluid Mech., 644, 433-463, 2010.

Babanin, A. V., Waseda, T., Kinoshita, T., and Toffoli, A.: Wave breaking in directional fields, J. Phys. Oceanogr., in press, 2011.

Bender III, L. C., Guinasso Jr., N. L., Walpert, J. N., and Howden, S. D.: A comparison of two methods for determining wave heights from a discuss buoy with a strapped-down accelerometer, Proc. 11th International Workshop on Wave Hindcasting and Forecasting and Coastal Hazard Symposium, Halifax, Canada, October, 18-23, 6 pp., 2009.

Battjes, J. A. and Janssen, J. P. F. M.: Energy loss and set-up due to breaking of random waves, Proc. 16th Int. Conf. Coastal Engineering, ASCE, 569-587, 1978.

Bidlot, J. R. Janssen, P. A. E. M., Hansen, B., and Günther, H.: A modified set up of the advection scheme in the ECMWF wave model, ECMWF Technical Memorandum, No. 237, ECMWF, Reading, 1997.

Booij, N., Ris, R. C., and Holthuijsen, L. H.: A third-generation wave model for coastal Regions 2: Verification, J. Geophys. Res.-Oceans, 104(C4), 7667-7682, doi:10.1029/1998JC900123, 1999.

Cavaleri, L.: Wave modeling - missing the peaks, J. Phys. Oceanogr., 39, 2757-2778, doi:10.1175/2009JPO4067.1, 2009.

Chiou, M. D., Chien, H., Kao, C. C., and Chuang, L. Z. H.: Comprehensive assessment on the measurement and snalysis of directional spectrum, Proc. of Sixth Int. Conf. on Costal and Port Eng. in Developing Countries (COPEDEC VI), Colombo, Sri Lanka, 2003.

Doong, D.-J., Chuang, L. Z. H., Kao, C. C., Lin, Y. B., and Jao, K. C.: Statistics of buoy-observed waves during typhoons at Taiwanese waters from 1997 to 2008, Proc. of OCEANS'09, Biloxi, USA, 2009.

Eldeberky, Y.: Nonlinear Transformation of Wave Spectra in the Nearshore Zone, Ph.D. thesis, TU-Deft, The Netherlands, 1996.

Fochesato, C., Grilli, S., and Dias, F.: Numerical modeling of extreme rogue waves generated by directional energy focusing, Wave Motion, 26, 395-416, 2007.

Greenslade, D. J. M.: A wave modelling study of the 1998 Sydney to Hobart yacht race, Aust. Meteorol. Mag., 50, 53-63, 2001.

Holthuijsen, L. H., Herman, A., and Booij, N.: Phase-decoupled refraction-diffraction for spectral wave models, Coast. Eng., 49, 291-305, 2003.

Hsiao, S. W.: Comparative study on the derivation of directional wave spectrum by finite Fourier Series Method and Maximum Likelihood Method, Master Dissertation, National Cheng Kung University, 1994 (in Chinese).

Hsu, T.-W., Ou, S.-H., and Liau, J.-M.: Hindcasting nearshore wind waves using a FEM code for SWAN, Coast. Eng., 52, 177-195, 2005. 
Huang, M. C. and Chen, J. Y.: Wave direction analysis from data buoys, Ocean Eng., 25, 621-637, 1998.

Isobe, M., Kondo, K., and Horikawa, K.: Extension of MLM for estimating directional wave spectrum, Proc. of the Symp. on Description and Modeling of Directional Seas, Paper No. A-6, 1984.

Janssen, T. T. and Herbers, T. H. C: Nonlinear wave statistics in a focal zone, J. Phys. Oceanogr., 39, 1948-1964, 2009.

Kao, C. C., Chang, Z. H., Lin, Y. P., and Lee, B. C.: An introduction to the operational Data Buoy System in Taiwan. Proc. of the Fourth Int. Conf. on the Mediterranean Coastal Environment (MEDCOAST 99), November 13-19, Turkey, 1999.

Kao, C. C., Chien, H., Chiou, M. D., and Chuang, Z. H.: Error analysis and correction on the directional wave spectrum derivation, China Ocean Engineering, 21, 24-33, 2003 (in Chinese).

Komen, G. J., Cavaleri, L., Donelan, M., Hasselmann, K., Hasselmann, S., and Janssen, P. A. E. M.: Dynamics and Modelling of Ocean Waves, Cambridge Univ. Press, Cambridge, UK, 532 pp., 1994.

Liu, P. C. and Babanin, A. V.: Using wavelet spectrum analysis to resolve breaking events in the wind wave time series, Ann. Geophys., 22, 3335-3345, doi:10.5194/angeo-22-3335-2004, 2004.

Liu, P. C., Chen, H. S., Doong, D.-J., Kao, C. C., and Hsu, Y.-J. G.: Monstrous ocean waves during typhoon Krosa, Ann. Geophys., 26, 1327-1329, doi:10.5194/angeo-26-1327-2008, 2008.

Onorato, M., Cavaleri, L., Fouques, S., Gramstad, O., Janssen, P. A. E. M., Monbaliu, J., Osborne, A. R., Pakozdi, C., Serio, M., Stansberg, C. T., Toffoli, A., and Trulsen, K.: Statistical properties of mechanically generated surface gravity waves: a laboratory experiment in a 3D wave basin, J. Fluid Mech., 637, 235-257, 2009a.

Onorato, M., Waseda, T., Toffoli, A., Cavaleri, L., Gramstad, O., Janssen, P. A. E. M., Kinoshita, T., Monbaliu, J., Mori, N., Osborne, A. R., Serio, M., Stansberg, C. T., Tamura, H., and Trulsen, K.: On the statistical properties of directional ocean waves: the role of the modulational instability in the formation of extreme events, Phys. Rev. Lett., 102, 114502, doi:10.1103/PhysRevLett.102.114502, 4 pp., 2009 b.
Ou, S.-H., Liau, J.-M., Hsu, T.-W., and Tzang, S.-Y.: Simulating typhoon waves by SWAN wave model in coastal waters of Taiwan, Ocean Eng., 29, 947-971, 2002.

Pierson, W. J., Donelan, M. A., and Hui, W. H.: Linear and nonlinear propagation of water wave groups, J. Geophys. Res., 97(C4), 5607-5621, 1992.

Rapp, R. J. and Melville, W. K.: Laboratory measurements of deepwater breaking waves, Philos. T. R. Soc. Lond. A, 311, 735-800, 1990.

Roland, A.: Development of the WWM II -Spectral Wave Modeling on Unstructured Meshes, Ph.D. thesis, Inst. for Hydraulic and Water Resources Eng., Univ. of Technology Darmstadt, 2009

Roland, A., Mewis, P., Zanke, U., Ou, S. H., Hsu, T. W., and Liau, J. M.: Verification and improvement of a spectral finite element wave model, in: Proc. Of Waves 2005, ASCE/COPRI, edited by: Billy Edge, The Fifth Int. Symp. on Wave Measurement and Analysis, Madrid, Spain, 2005.

Roland, A., Cucco, A., Ferrarin, C., Hsu, T.-W., Liau, J.-M., Ou, S.-H., Umgiesser, G., and Zanke, U.: On the development and verification of a 2-D coupled wave-current model on unstructured meshes, J. Marine Syst., 78, Supplement 1, S244S254, doi:10.1016/j.jmarsys.2009.01.026, 2009.

Schwartz, L. W. and Fenton, J. D.: Strongly nonlinear waves, Annu. Rev. Fluid Mech., 14, 39-60, 1982.

Tolman, H. L.: User manual and system documentation of WAVEWATCH-III version 2.22, NOAA/NWS/NCEP/MMAB Technical Note 222, 133 pp., 2002.

Vincent, C. L. and Briggs, M. J.: Refraction-diffraction of irregular waves over a mound, J. Waterw. Port C. Div.-ASCE, 115(2), 269-284, 1989.

Waseda, T., Kinoshita, T., and Tamura, H.: Evolution of a random directional wave and freak wave occurrence, J. Phys. Oceanogr., 39, 621-639, 2009.

Yanenko, N. N.: The Method of Fractional Steps, Springer, Berlin, 1971.

Zijlema, M.: Computation of wind-wave spectra in coastal waters with SWAN on unstructured grids, Coast. Eng., 57, 267-277, 2010 . 